\title{
Mitochondrial Parkin Recruitment Is Impaired in Neurons Derived from Mutant PINK1 Induced Pluripotent Stem Cells
}

\author{
Philip Seibler, ${ }^{1,2}$ John Graziotto, ${ }^{1}$ Hyun Jeong, ${ }^{1}$ Filip Simunovic, ${ }^{1,3}$ Christine Klein, ${ }^{2}$ and Dimitri Krainc ${ }^{1}$ \\ ${ }^{1}$ Department of Neurology, Massachusetts General Hospital, Harvard Medical School, MassGeneral Institute for Neurodegenerative Disease, Charlestown, \\ Massachusetts $02129,{ }^{2}$ Section of Clinical and Molecular Neurogenetics at the Department of Neurology, University of Lübeck, 23562 Lübeck, Germany, and \\ ${ }^{3}$ Mediterranean Institute for Life Sciences, Split 21000, Croatia
}

Genetic Parkinson disease (PD) has been associated with mutations in PINK1, a gene encoding a mitochondrial kinase implicated in the regulation of mitochondrial degradation. While the studies so far examined PINK1 function in non-neuronal systems or through PINK1 knockdown approaches, there is an imperative to examine the role of endogenous PINK1 in appropriate human-derived and biologically relevant cell models. Here we report the generation of induced pluripotent stem (iPS) cells from skin fibroblasts taken from three PD patients with nonsense (c.1366C > T; p.Q456X) or missense (c.509T > G; p.V170G) mutations in the PINK1 gene. These cells were differentiated into dopaminergic neurons that upon mitochondrial depolarization showed impaired recruitment of lentivirally expressed Parkin to mitochondria, increased mitochondrial copy number, and upregulation of PGC-1 $\alpha$, an important regulator of mitochondrial biogenesis. Importantly, these alterations were corrected by lentiviral expression of wild-type PINK1 in mutant iPS cell-derived PINK1 neurons. In conclusion, our studies suggest that fibroblasts from genetic PD can be reprogrammed and differentiated into neurons. These neurons exhibit distinct phenotypes that should be amenable to further mechanistic studies in this relevant biological context.

\section{Introduction}

Parkinson disease (PD) is the second most common chronic progressive neurodegenerative disorder, and the clinical features of PD likely result from the loss of dopaminergic (DA) neurons in the substantia nigra pars compacta (Fahn, 2003). Although "idiopathic" $\mathrm{PD}$ is more common, $\sim 2-3 \%$ of PD cases can currently be linked to a single genetic factor, and over the last several years, the discovery of these genes has provided insights into the cellular and molecular pathogenesis of PD (Gasser, 2007; Klein and Schlossmacher, 2007). A clinical syndrome closely resembling "idiopathic" PD has been reported for recessively inherited Parkin (Kitada et al., 1998) and PTEN-induced putative kinase 1 (PINK1) (Valente et al., 2004) mutations, suggesting that pathways uncovered in these monogenic forms of PD may play a direct role in the etiology of the more common sporadic disorder. Recent studies demonstrated the involvement of both Parkin and PINK1 in mitochondrial function (Narendra et al., 2010; Vives-Bauza et al., 2010). Parkin is localized in the cytosol and functions as an E3 ubiquitin ligase, whereas the mitochondrial kinase PINK1 is localized on the outer mitochondrial membrane. Interestingly, Parkin is translocated to damaged mitochondria (Narendra et al., 2008) in a PINK1-dependent manner,

Received Aug. 24, 2010; revised Dec. 20, 2010; accepted Jan. 17, 2011.

This work was supported by R01NS051303 (D.K.), the German Academic Exchange Service (P.S.), and the Fritz Thyssen Foundation (C.K.). C.K. is a recipient of a career development award from the Volkswagen Foundation and from the Hermann and Lilly Schilling Foundation. We thank George Daley (Children's Hospital, Boston, MA) for providing retroviral reprogramming constructs, Laurence Daheron (Massachusetts General Hospital, Boston, MA) for helpful discussions and experimental advice, and Stuart Chambers and Lorenz Studer (Sloan-Kettering Institute, New York, NY) for helpful technical advice.

Correspondence should be addressed to Dr. Dimitri Krainc, Massachusetts General Hospital, 114 16th Street, Room 2008, Charlestown, MA 02129. E-mail: krainc@helix.mgh.harvard.edu or dkrainc@partners.org.

DOI:10.1523/JNEUROSCI.4441-10.2011

Copyright $\odot 2011$ the authors $\quad 0270-6474 / 11 / 315970-07 \$ 15.00 / 0$ indicating that PINK1 functions upstream from Parkin in a common pathway (Narendra et al., 2010; Rakovic et al., 2010; VivesBauza et al., 2010). While these studies were performed with knockdown of PINK1 or in non-neuronal cell types, we used induced pluripotent stem (iPS) cells, derived through a reprogramming mechanism from human somatic cells (Takahashi et al., 2007), to examine the role of endogenous PINK1 in patient-derived dopaminergic neurons.

\section{Materials and Methods}

Cell culture and infection. Primary human dermal fibroblast and irradiated mouse embryonic fibroblast (MEF; GlobalStem) cells were cultured in fibroblast medium: DMEM supplemented with 10\% FBS (Hyclone), L-glutamine, sodium pyruvate, nonessential amino acids, and penicillin/streptomycin.

Human iPS cells were maintained on irradiated MEF feeder layers in iPS cell medium: DMEM/F12 (StemCell Technologies) with 20\% knock-out serum replacement, L-glutamine, nonessential amino acids, $\beta$-mercaptoethanol, $10 \mathrm{ng} / \mathrm{ml} \mathrm{bFGF}$, and penicillin/streptomycin. IPS cell colonies were passaged enzymatically using Collagenase type IV. All cells were maintained at $37^{\circ} \mathrm{C}$ in a saturated humidity atmosphere containing $5 \% \mathrm{CO}_{2}$.

Human Parkin and PINK1-V5 cDNA were cloned into lentiviral pER4 vectors with neuron-specific PGK promoter. Virus production was conducted according to standard protocols for a virus titer of $4.2 \times 10^{5}$ infectious particles (i.p.)/ $/ \mu$ l (Parkin virus) and $9.4 \times 10^{5}$ i.p. $/ \mu \mathrm{l}$ (PINK1-V5 virus). Cells were infected on day 25 of differentiation with a multiplicity of infection of 1-3. Infection efficiencies of Parkin virus (70\%) and PINK1-V5 virus (90\%) were analyzed by immunostaining $5 \mathrm{~d}$ after infection (see Fig. 3B).

Reprogramming. For the generation of iPS cells, primary human dermal fibroblasts were transduced by OCT4, SOX2, cMYC, and KLF4. Retroviral pMIG vectors containing the four factors were obtained from 

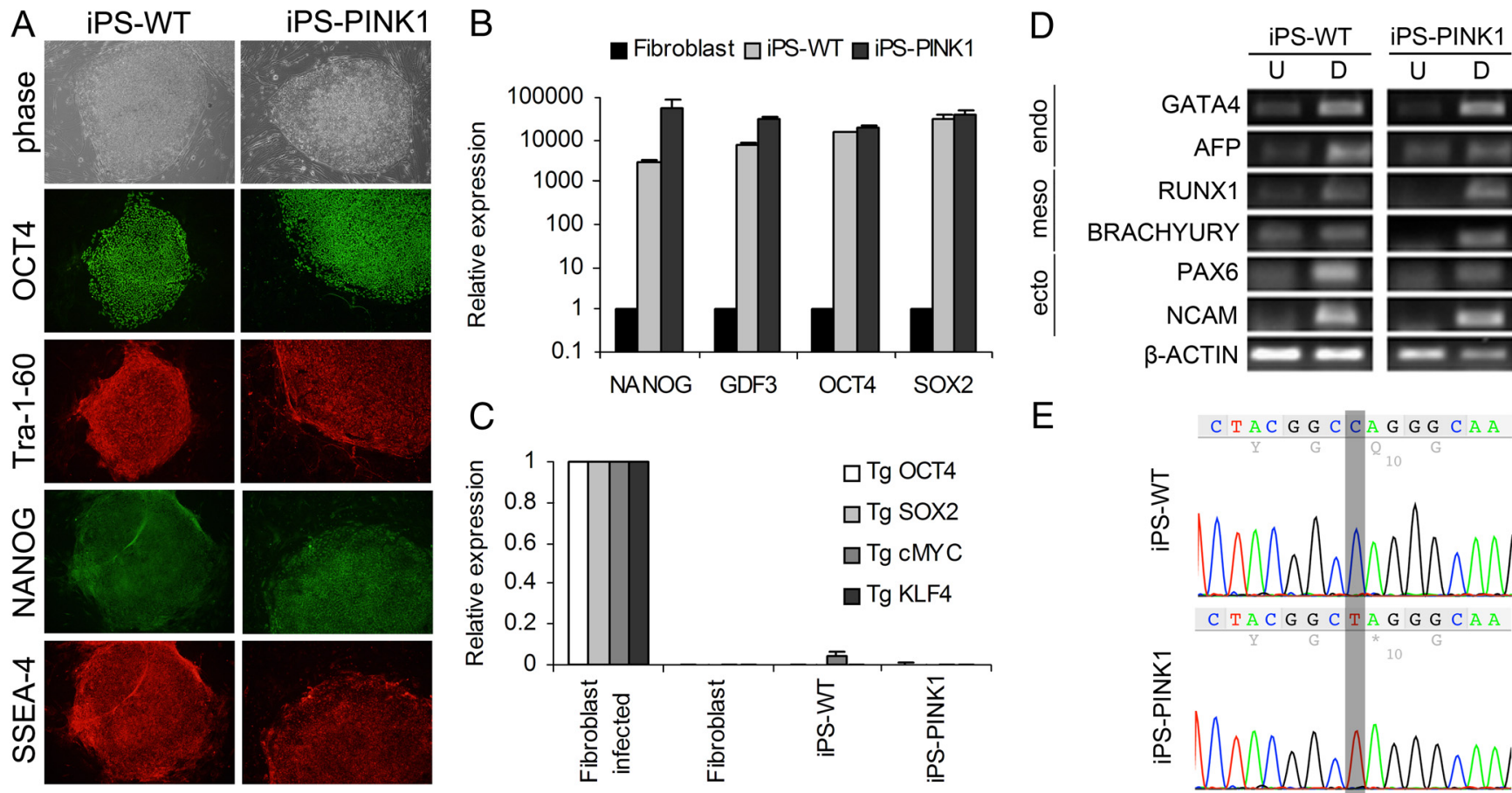

$E$

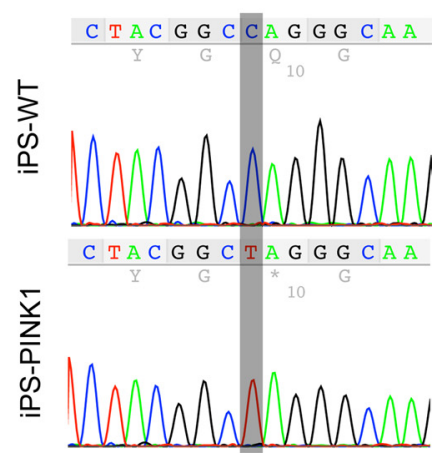

Figure 1. Generation of iPS cells from a PD patient harboring a PINK1 mutation and a healthy control individual. IPS-PINK1 was established from a PD patient with mutant PINK1 (c.1366C $>$ T) and iPS-WT from a healthy family member. $\boldsymbol{A}$, Immunofluorescence analysis shows presence of pluripotency markers OCT4, Tra-1-60, NANOG, and SSEA-4. $\boldsymbol{B}$, Expression levels of pluripotency markers NANOG, GDF3, OCT4, and SOX2 in fibroblasts and iPS cell lines relative to $\beta$-actin (a loading control) as assessed by quantitative RT-PCR. The values from parental fibroblasts were set to 1 . C, Residual expression levels of transgenes OCT4, SOX2, CMYC, and KLF4 (relative to $\beta$-actin) were examined by quantitative RT-PCR. The values from the infected fibroblasts (isolated $7 \mathrm{~d}$ after infection) were set to 1. Uninfected fibroblasts were used as negative controls. The error bars indicate SD. D, RT-PCR analyses of various differentiation markers for the three germ layers (endoderm: GATA4, AFP; mesoderm: RUNX1, BRACHYURY; ectoderm: PAX6, NCAM) in iPS cells that were undifferentiated (U) and after $4 \mathrm{~d}$ in suspension culture followed by $7 \mathrm{~d}$ in adherent culture (D). $E$, Direct sequencing confirmed the PINK1 mutation c.1366C >T (p.0456X; nonsense mutation) in iPS-PINK1.

the G. Q. Daley laboratory (Children's Hospital, Boston, MA) and viral production was conducted as previously described (Park et al., 2008a). All four vectors contain a GFP sequence, and the concentrated virus was titered by determining the percentage of infected 293FT HEK cells using FACS as reported previously (Tiscornia et al., 2006). On day 0, fibroblast cells $\left(3 \times 10^{5}\right)$ were plated in a $6 \mathrm{~cm}$ dish and infected on day 1 with an infection efficiency of $\sim 80 \%$. After $48 \mathrm{~h}$, the cells were trypsinized and replated onto two gelatin-coated $10 \mathrm{~cm}$ dishes in fibroblast medium. Cells were cultured in iPS cell media starting from day 4 and treated with $0.5 \mathrm{~mm}$ valproic acid (EMD Biosciences) for 1 week. To establish iPS cell lines, iPS cell colonies were picked $\sim 1$ month after infection based on their stem-cell-like morphology and the silencing of the retroviral GFP signal. The picked colonies were subsequently expanded and maintained on MEF feeder layers in iPS cell media. Karyotype analysis was performed at Cell Line Genetics using standard protocols for high-resolution G-banding. Spontaneous differentiation through embryoid body (EB) formation was performed as described before (Xu et al., 2001).

Neuronal in vitro differentiation. Directed differentiation in vitro toward DA neurons was conducted as described previously (Chambers et al., 2009), with minor modifications as follows: IPS cell colonies were dissociated into single cells using Accutase and replated onto Matrigelcoated dishes in mTeSR1 medium (StemCell Technologies), supplemented with ROCK inhibitor Y-27632 (EMD Biosciences) at a density of $35,000 \mathrm{cells} / \mathrm{cm}^{2}$. Differentiation was started at a confluence of $\sim 80 \%$ by adding knock-out serum replacement medium containing SB431542 (Tocris Bioscience) and Noggin (R\&D Systems). Upon day 5 of differentiation, increasing amounts of $\mathrm{N} 2$ medium was added $(25 \%, 50 \%, 75 \%)$ to knock-out serum replacement medium every $2 \mathrm{~d}$ as reported (Chambers et al., 2009). On day 11 of differentiation, cells were passaged en bloc (size of 1-2 mm) and plated onto coated glass coverslips (Assistent) in N2 medium. Glass coverslips were incubated in nitric acid for $24 \mathrm{~h}$, washed with water and ethanol, incubated overnight in $75 \mu \mathrm{g} / \mathrm{ml}$ poly-D-lysine (Sigma), washed with water, and incubated overnight in $5 \mu \mathrm{g} / \mathrm{ml} \mathrm{laminin}$ (Roche). Patterning of cells into the DA lineage was initiated by adding BDNF, ascorbic acid,
SHH, and FGF8a. After 1 week of culture, cells were differentiated in the presence of BDNF, ascorbic acid, GDNF, TGF- $\beta 3$, and cyclic-AMP as previously reported (Perrier et al., 2004; Chambers et al., 2009).

Immunofluorescence and cell counts. IPS cells and differentiated neurons were grown on glass coverslips, fixed in $4 \%$ formaldehyde for 15 min, permeabilized, and blocked with $0.1 \%$ Triton X-100 in $4 \%$ appropriate normal serum in PBS for $1 \mathrm{~h}$. Immunofluorescence staining was performed using primary antibodies against OCT4 (1:400; Abcam), Tra1-60 (1:400; Millipore), Nanog (1:100; Stemgent), SSEA-4 (1:200; Millipore), TUJ1 (1:1000; Covance), tyrosine hydroxylase (TH) (1:400; Calbiochem and 1:100; Novus Biologicals), Parkin (1:200; Santa Cruz Biotechnology), TOM20 (1:200; Santa Cruz Biotechnology), and anti-V5 (1:500; Invitrogen). Appropriate secondary antibodies were obtained from Invitrogen. Quantification of immunofluorescence staining was performed on fields of neuronal monolayer cultures (supplemental Fig. S2 $D$, available at www.jneurosci.org as supplemental material) acquired at $20 \times$ magnification (dimensions: $4080 \times 3072$ ). Aggregated clusters of neurons were excluded from counts. Each field was scored first for DAPI (4',6-diamidino-2-phenylindole)-positive nuclei followed by TUJ1 and, subsequently, TH colocalization. The total number of cells analyzed from two independent differentiation experiments exceeded 12,000 cells.

Confocal microscopy. Differentiated neurons were treated with the potassium ionophore valinomycin ( $1 \mu \mathrm{M}$, Sigma) for $12 \mathrm{~h}$. Colocalization imaging of Parkin translocation was performed on fixed samples using confocal microscopy (TCS SL; Leica Microsystems) with the $63 \times / 1.4$ oil HCX PL APO objective. The quantitative colocalization analysis of Parkin and Tom 20 signals was performed with ImageJ and JACoP plug-in (Bolte and Cordelières, 2006) to determine Manders' coefficient (Manders et al., 1992) from 0 to $1(0=$ non-overlapping images and $1=$ colocalized images $)$. The coefficient displays the ratio of the summed intensities of pixels from the Parkin image for which the intensity in the Tom 20 image is above zero to the total intensity in the Parkin image. Therefore, Manders' coefficient is a good indicator of the proportion of the Parkin signal coincident with a signal of Tom 20 over its total intensity. 
RNA and DNA extraction and PCR analysis. Total RNA from iPS cells and plated EBs was prepared by using the RNeasy kit (Qiagen) according to the manufacturer's instructions and then reverse transcribed into cDNA with the SuperScript III First-Strand Synthesis System (Invitrogen). Quantitative RT-PCR was performed with SYBR GreenER (Invitrogen) on the iCycler system (Bio-Rad). Genomic DNA was isolated from cells using DNeasy kit (Qiagen). Primers used in this study can be found in supplemental Table S1 (available at www. jneurosci.org as supplemental material).

Western blotting. Neuronal cultures were scraped and proteins from cell pellet were extracted using SDS extraction buffer. Antibodies used for Western blotting were anti-TH (1:1000; Calbiochem) and anti- $\alpha$-Tubulin (1:6000; Sigma). Detection was done using the Odyssey infrared imaging system (LI-COR Biosciences).

\section{Results}

Generation of iPS cells from PD patients harboring PINK1 mutations

For the production of two iPS cell lines, we used primary human dermal fibroblast cultures with wild-type (WT) and mutant PINK1, both recruited from a German family (Hedrich et al., 2006). The control fibroblast line was established from a healthy family member of the PD patient without the PINK1 mutation. The mutation carrier exhibited the onset of clinical symptoms at the age of 53 years characterized as bradykinesia, rigidity, postural instability, and good response to levodopa treatment. The homozygous PINK1 nonsense mutation, c.1366C $>$ T (p.Q456X), leads to a premature stop codon and reduction in PINK1 mRNA levels by $80-$ $90 \%$ compared to the PINK1 mRNA levels in controls, most likely due to nonsense-mediated mRNA decay (Grünewald et al., 2007).

The reprogramming was initiated by transduction of the four factors OCT4, SOX2, KLF4, and cMYC via retrovirus into the genome of the fibroblasts (Park et al., 2008a). A histone deacetylase inhibitor, valproic acid, was included in the cocktail to increase the efficiency of reprogramming (Huangfu et al., 2008). After a month of culturing, iPS cell colonies were picked based on their characteristic embryonic stem-cell-like morphology and silencing of the retroviral GFP signal (Yao et al., 2004). Two colonies from the wild-type and three from the PINK1 mutant line gave rise to stable iPS cell lines that were further expanded on mouse embryonic fibroblasts (MEFs).

We next examined the colonies from iPS-PINK1 and iPS-WT lines for pluripotency (Fig. 1). Immunofluorescence staining showed high expression of pluripotency markers OCT4, Tra-1-60, NANOG, and SSEA-4 (Fig. $1 \mathrm{~A}$ ), and quantitative RT-PCR analysis confirmed a dramatic increase of pluripotency marker genes in iPS lines compared with the parental fibroblast cells (Fig. $1 B$ ). The expression of viral transgenes OCT4, SOX2, KLF4, and cMYC was efficiently silenced as determined by quantitative RT-PCR (Fig. 1C). We next examined the differentiation ca- pacity of the iPS cells in vitro through the formation of EBs. Spontaneous differentiation into derivatives of the three embryonic germ layers was confirmed after $11 \mathrm{~d}$ of culture (Fig. $1 D)$. The iPS cells were karyotypically normal (supplemental Fig. S1, available at www.jneurosci.org as supplemental material), and direct sequencing detected the PINK1 mutation in the patient-derived iPS cells (Fig. $1 E$ ). Together, these data indicated successful reprogramming of WT and mutant PINK1 fibroblasts to a pluripotent state.

\section{Generation of human DA neurons from WT and mutant PINK1 iPS cells}

We next differentiated the two iPS cell lines into DA neurons using a protocol that achieves rapid neuronal conversion (25-30 d) on adherent cell culture (Chambers et al., 2009). Traditional EB formation (30-50 d) was found to be inefficient for neural differentiation from iPS cell cultures, and stromal feeders could contribute undefined factors to the cell culture (Chambers et al., 2009).

In the first step, undifferentiated iPS cell colonies were dissociated into single cells and replated onto Matrigel-coated dishes 
A

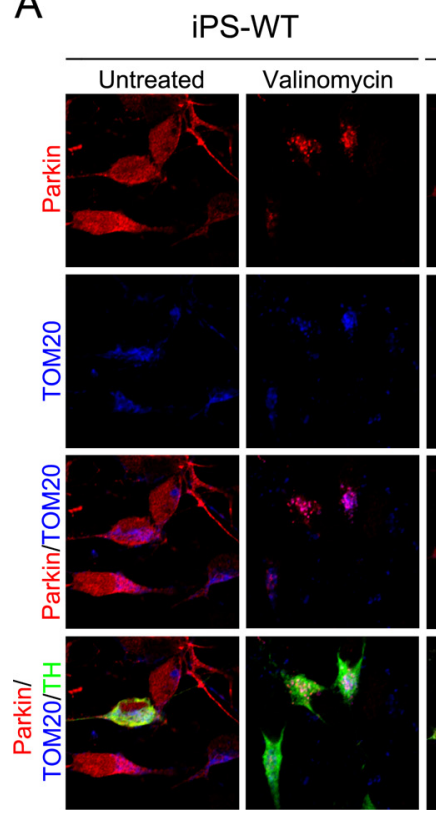

iPS-PINK1 mutants

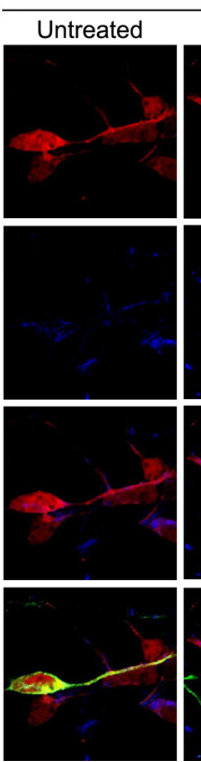

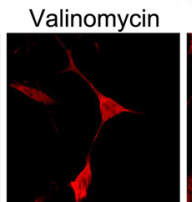
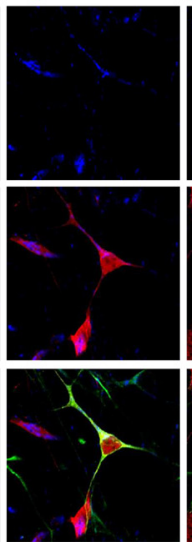

iPS-PINK1 mutants + PINK1-WT

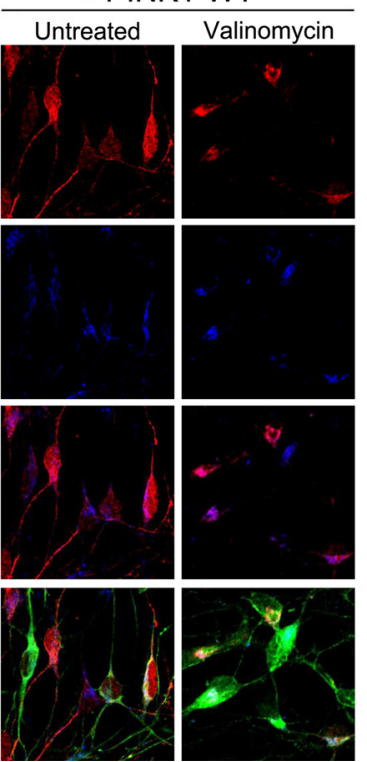

B
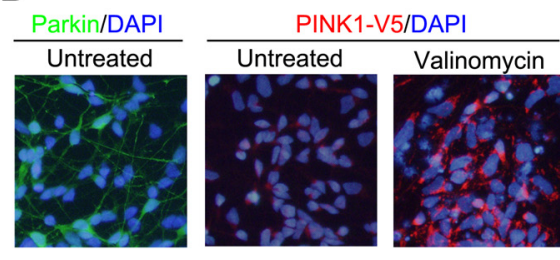

C

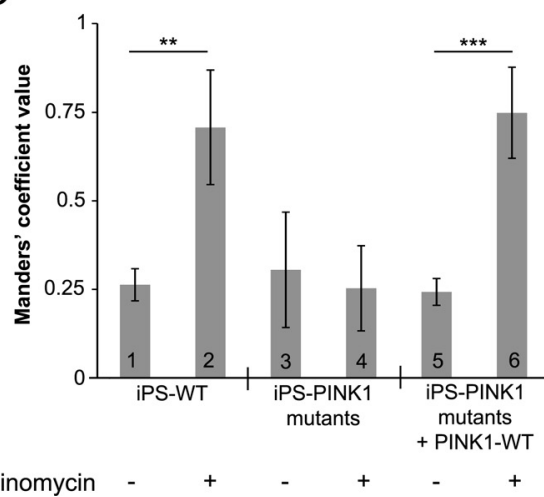

Figure 3. Stress-induced mitochondrial translocation of Parkin is impaired in mutant PINK1 iPS cell-derived human DA neurons. A, Neuronal cultures were infected with WT Parkin and treated with $1 \mu \mathrm{m}$ valinomycin or vehicle for $12 \mathrm{~h}$. Cells were fixed and immunostained with antibodies against Parkin (red), mitochondrial marker TOM20 (blue), and the DA marker TH (green). Parkin colocalizes with mitochondria in control DA neurons (iPS-WT, left panel) but not in mutant PINK1 neurons (iPS-PINK1 mutants, middle panel). Lentiviral transduction of mutant PINK1 neurons with WT PINK1 restored Parkin translocation (right panel). B, Validation of lentiviral Parkin and PINK1-V5 infection of iPS cell-derived neurons. Neuronal culture was infected with lenti-Parkin lentivirus (left panel), or lenti-PINK-V5 (middle and right panels), coimmunostained with anti-Parkin antibody (green), anti-V5 antibody (red), and DAPI (blue). PINK1 is synthesized as a full-length form $(\sim 66 \mathrm{kDa})$ that is proteolytically processed upon mitochondrial entry to its cleaved $\sim 55 \mathrm{kDa}$ form. In untreated neuronal culture C-terminal V5-tag is cleaved and only a few cells are PINK1-V5positive (middle panel). Treatment with $1 \mu \mathrm{m}$ valinomycin for $12 \mathrm{~h}$ results in PINK1-V5 accumulation in its uncleaved full-length form ( $\sim 66 \mathrm{kDa}$; right panel). The infection efficiency was $70-90 \%$. C, The quantitative colocalization analysis of Parkin and Tom20 signals was performed with ImageJ and JACOP plug-in (Bolte and Cordelières, 2006) to determine Manders' coefficient (Manders et al., 1992) from 0 to 1 ( 0 = non-overlapping images and 1 = colocalized images). The highest values were found for valinomycin-treated iPS-WT (sample 2) and PINK1-WT-infected iPS-PINK1 mutants (sample 6), suggesting a translocation of Parkin to mitochondria in the presence of a functional PINK1 protein. The results were analyzed with an unpaired $t$ test with error bars indicating $\mathrm{SD}, n=4$ (samples 1, 2: $p=0.002$; samples 5, 6: $p=0.0003$ ).

in mTeSR1 medium, supplemented with a ROCK inhibitor (Y27632). At a cell density of $\sim 80 \%$, differentiation was started by changing the medium to knock-out serum replacement medium containing SMAD inhibitors Noggin and SB431542 (Chambers et al., 2009). The blockade of SMAD signaling has been shown to help destabilize pluripotency while promoting neuralization of primitive ectoderm (Lee et al., 2007; Xu et al., 2008). By day 11 of culture, a dense layer of cells (supplemental Fig. S2 A, available at www.jneurosci.org as supplemental material) was mechanically isolated and passaged en bloc. To push the differentiating precursor cells toward a DA state, the cells were exposed to a number of sequential factors, including BDNF, ascorbic acid, and the morphogenic factors sonic hedgehog $(\mathrm{SHH})$ and FGF8a, as reported previously (Chambers et al., 2009) and allowed to differentiate for a total of 1 week. During this period of differentiation, neuronal precursor cells were seen migrating out from the bloc. By day 16 of differentiation, the formation of neural rosette structures was observed (supplemental Fig. S2 B, available at www. jneurosci.org as supplemental material), indicating the presence of immature CNS cells. Terminal differentiation was induced by replacement of factors SHH and FGF8a with GDNF, TGF- $\beta 3$, and cyclic-AMP (Chambers et al., 2009). Neurogenesis was largely completed by day 30 of differentiation with a large proportion of neurons growing in a monolayer (supplemental Fig. $\mathrm{S} 2 C$, available at www.jneurosci.org as supplemental material). WT and mutant PINK1 neurons were grown simultaneously until day 60 of differentiation.

Immunofluorescence staining (Fig. $2 A$ ) displayed that $\sim 60 \%$ of the total cells (Fig. $2 B$ ) derived from iPS-WT and iPS-PINK1 expressed the neuron-specific marker neuronal class III $\beta$-Tubulin (TUJ1) (iPS-WT, $62 \pm 15 \%$; iPS-PINK1, $66 \pm 16 \%$ ). The percentage of neurons coexpressing TUJ1 and tyrosine hydroxylase, $\mathrm{TH}$, the rate-limiting enzyme in the synthesis of DA, was slightly higher in iPS-PINK1 $(16 \pm 1 \%)$ compared to iPS-WT $(11 \pm 3 \%)$. Expression of TH was confirmed by Western blotting (Fig. 2C). Most of the remaining cells were Nestinpositive neuronal precursor cells that did not differentiate into mature neurons (data not shown). As expected, levels of PINK1 were reduced in mutant neuronal cultures (Fig. 2D).

\section{Stress-induced mitochondrial translocation of Parkin is impaired in mutant PINK1 iPS cell-derived human DA neurons}

While previous studies reported Parkin recruitment to depolarized mitochondria in animal cells, immortalized human cell lines, and human PD patient's fibroblasts (Narendra et al., 2010; Rakovic et al., 2010; Vives-Bauza et al., 2010), we asked whether the Parkin/PINK1 interaction is impaired in human dopaminergic neurons that contain an endogenous PINK1 mutation. To address this question, we treated neurons derived from our iPS-WT and iPS-PINK1 lines with the potassium ionophore valinomycin, which triggers loss of mitochondrial membrane potential and induces mitochondrial translocation of Parkin in PD patient fibroblasts (Rakovic et al., 2010). Since the anti-Parkin antibody was not sensitive enough for immunostaining of endogenous Parkin, we cloned WT Parkin into a lentiviral construct and transduced our iPS cell-derived neurons. To exclude variations due to reprogramming and differentiation, we examined Parkin translocation in two addi- 
tional iPS cell lines derived from PINK1 PD patients. IPSPINK1-2 (supplemental Fig. S3, available at www.jneurosci.org as supplemental material) was derived from a family member carrying the same nonsense mutation (c.1366C $>$ T; p.Q456X) and iPS-PINK1-3 (supplemental Fig. S4, available at www. jneurosci.org as supplemental material) was generated from a patient of another family harboring a missense mutation in the PINK1 gene (c.509T > G; p.V170G). These patients exhibited the onset of clinical symptoms at the ages of 61 and 31, respectively.

Under basal conditions, Parkin was localized in the cytosol and the neurites of DA neurons in both control (Fig. 3A, left) and mutant PINK1 (Fig. 3A, middle) cells. However, treatment with valinomycin resulted in dramatic mitochondrial translocation of Parkin in the control but not in the PINK1 mutant neurons (Fig. $3 \mathrm{~A}$, left, middle). These results suggested that in the presence of endogenous PINK1 mutation, Parkin did not translocate to mitochondria even when moderately overexpressed by lentiviral transduction. To determine whether expression of WT PINK1 could rescue this phenotype, we cloned WT PINK1-V5 into a lentiviral construct and infected iPS-derived mutant PINK1 neurons. High transduction efficiency $(70-90 \%)$ was achieved for both Parkin and PINK1 constructs, as determined by immunostaining (Fig. 3B). Full-length PINK1 $(\sim 66 \mathrm{kDa})$ is proteolytically processed upon entry into mitochondria to its cleaved $\sim 55$ $\mathrm{kDa}$ form (Weihofen et al., 2008). Therefore, PINK1 C-terminal V5-tag gets normally cleaved under basal conditions and expression of transduced PINK1 cannot be detected (Fig. 3B, middle). Upon treatment with $1 \mu \mathrm{M}$ valinomycin for $12 \mathrm{~h}$, PINK1-V5 accumulated in its uncleaved full-length form $(\sim 66 \mathrm{kDa})$, which permits its detection (Fig. 3B, right) (Narendra et al., 2010). This approach revealed that overexpression of WT PINK1 in PINK1 mutant neurons restored the translocation of Parkin to mitochondria (Fig. $3 A$, right). This effect was observed in neurons derived from all three PINK1 mutant iPS lines (supplemental Fig. S5, available at www.jneurosci.org as supplemental material). Colocalization images shown in Figure 3 were quantified with ImageJ software (Fig. $3 C$ ). Together, these results demonstrate that endogenous PINK1 mediates mitochondrial translocation of Parkin in human dopaminergic neurons.

Since previous studies suggested that Parkin translocation to mitochondria participates in autophagic degradation of dysfunctional mitochondria (Narendra et al., 2010; Rakovic et al., 2010; Vives-Bauza et al., 2010), we investigated whether PINK1 deficiency affects mitochondrial DNA (mtDNA) copy number. Using quantitative RT-PCR for mtDNA (Parfait et al., 1998), we observed a reduction in mtDNA in the iPS-WT, but not in the PINK1 mutant cells upon mitochondrial depolarization (Fig. $4 A$ ). Importantly, overexpression of WT-PINK1 in the mutant line resulted in a comparable effect of mitochondrial depolarization on mtDNA as observed in WT cells (Fig. 4A). These results suggested that deficient translocation of Parkin to mitochondria in mutant PINK1 neurons may lead to accumulation of mitochondria due to their decreased clearance. Since the increase in mtDNA can be also due to increased mitochondrial production, we investigated the expression levels of PGC- $1 \alpha$, an important inducer of mitochondrial biogenesis (Wu et al., 2006; Aquilano et al., 2010). Interestingly, PGC- $1 \alpha$ levels significantly increased in mutant PINK1 but not in WT neurons upon mitochondrial depolarization, and this effect was abolished by lentiviral WTPINK1 expression in mutant PINK1 cells (Fig. 4B). Together, these results suggested that decreased translocation of Parkin to mitochondria in mutant PINK1 lines leads to increased mito-
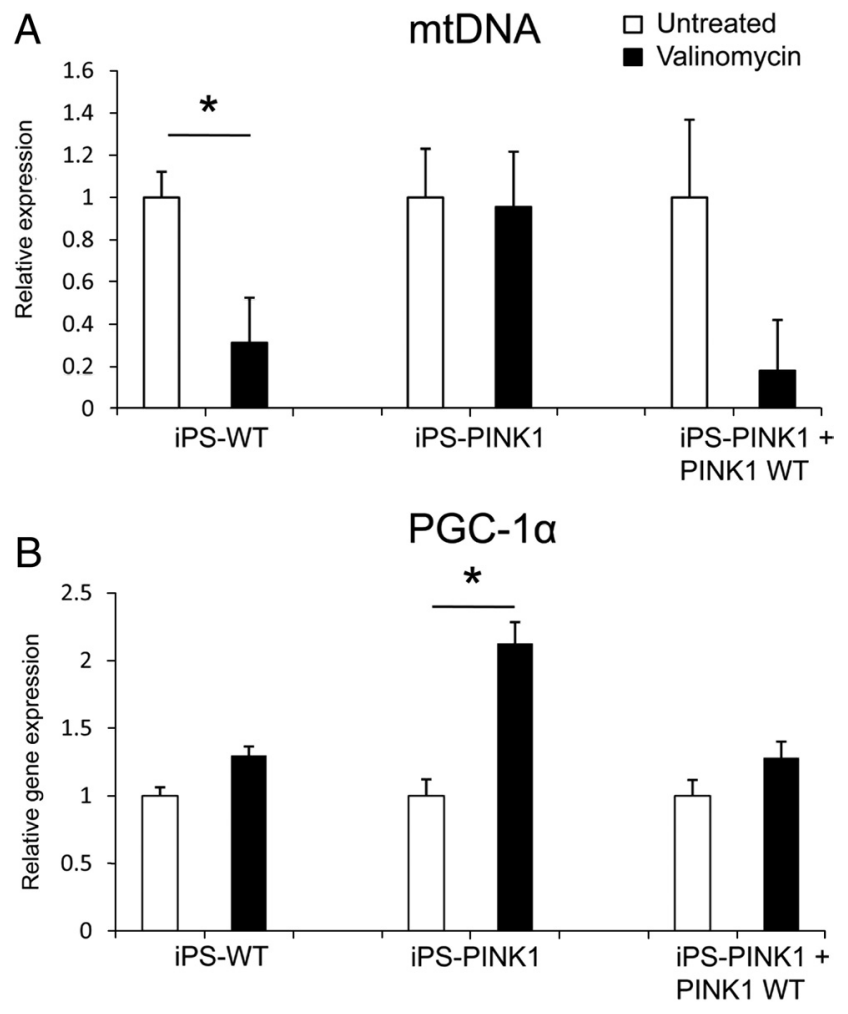

Figure 4. Abnormalities in mtDNA copy number and PGC-1 $\alpha$ expression in mutant PINK1 iPS neurons. Neurons were either untreated or treated with $1 \mu \mathrm{m}$ valinomycin for $12 \mathrm{~h}$. $\boldsymbol{A}$, Upon treatment, there is significant reduction in mtDNA copy number in the iPS-WT and mutant iPS-PINK1 neurons transduced with wt-PINK1, but not in the PINK1 mutant line. $\boldsymbol{B}, \mathrm{PGC}-1 \alpha$ gene expression is significantly upregulated after valinomycin treatment in the PINK1 mutant line but not in the WT or lenti-PINK1-expressing mutant line. Results in $\boldsymbol{A}$ and $\boldsymbol{B}$ are shown as fold change relative to untreated samples and normalized to $\beta$-actin. The results were analyzed with an unpaired $t$ test, with error bars representing SEM (mtDNA untreated vs treated: $p<$ 0.005; PGC $-1 \alpha$ untreated vs treated: $p=0.02 ; n=3$ ).

chondrial copy number and increased expression of PGC- $1 \alpha$. It will be of interest to determine whether this increase in PGC- $1 \alpha$ represents a compensatory response of mutant PINK1 neurons to restore mitochondrial function.

\section{Discussion}

Recent reports have demonstrated that iPS cells can be generated from patients and subsequently differentiated into the disease relevant cell types (Dimos et al., 2008; Park et al., 2008b; Ebert et al., 2009; Soldner et al., 2009). For neurological disorders, neurons have been successfully differentiated from fibroblasts of patients with amyotrophic lateral sclerosis (Dimos et al., 2008), spinal muscular atrophy (SMA) (Ebert et al., 2009), and familial dysautonomia (FD) (Lee et al., 2009). Of these, neurons derived from the SMA patient and the FD patient exhibited discernable phenotypes. While iPS cells derived from sporadic PD patients have been differentiated into DA neurons, no phenotype has been observed in these neurons (Soldner et al., 2009). Since genetic PD has not been studied using this system, we reprogrammed fibroblasts from patients with PINK1-linked PD and genetically matched normal subjects. We found that the mutation did not affect the ability of patient fibroblasts to be induced into iPS cells. Furthermore, patient-derived iPS cells were able to differentiate into DA neurons with efficiency comparable to the control iPS cells. 
We established these DA neurons as a model to begin to study the role of the endogenous PINK1 mutation in a more relevant cell type for PD. PINK1 is a mitochondrial kinase that protects cells against mitochondrial stress (Wood-Kaczmar et al., 2008), whereas loss of PINK1 has been linked to increased levels of oxidative stress (Hoepken et al., 2007) and loss of the mitochondrial membrane potential (Gegg et al., 2009). Previous studies established the importance of PINK1 in Parkin recruitment to mitochondria and in mitochondrial function but relied on PINK1 knockdown models or were performed in non-neuronal systems (Narendra et al., 2010; Rakovic et al., 2010; Vives-Bauza et al., 2010). Here we demonstrate that the presence of endogenous mutant PINK1 in human DA neurons leads to diminished recruitment of Parkin to mitochondria. Importantly, expression of normal PINK1 rescued the effect of mutant PINK1 on Parkin translocation, further validating the relevance of PINK1/Parkin pathway in human neurons. In addition, we found that mtDNA does not decrease in depolarized mutant PINK1 neurons as it does in WT neurons. This increase in mtDNA may reflect the impaired degradation of depolarized mitochondria due to PINK1 deficiency as suggested previously (Vives-Bauza et al., 2010). Interestingly, we also found upregulation of PGC- $1 \alpha$ in depolarized mutant PINK1 neurons, suggesting increased mitochondrial biogenesis in mutant cells. PGC1 $\alpha$ has been implicated in the pathogenesis of several neurodegenerative disorders, including PD (Cui et al., 2006; Finck and Kelly, 2006; Weydt et al., 2006; Qin et al., 2009; Zheng et al., 2010), but its precise role in $\mathrm{PD}$ remains unknown. We hypothesize that the increase in PGC- $1 \alpha$ expression in mutant PINK1 neurons reflects a compensatory response to restore deficient mitochondrial function in the presence of mutant PINK1. However, further studies are required to examine mitochondrial function in PINK1 and Parkin human neurons and clarify the role of PGC- $1 \alpha$ in this context.

In summary, our study demonstrates that it is possible to reprogram and differentiate fibroblasts from genetic PD to investigate and validate the function of endogenous mutations, and suggests that this approach could be used for the studies of PD pathogenesis.

\section{References}

Aquilano K, Vigilanza P, Baldelli S, Pagliei B, Rotilio G, Ciriolo MR (2010) Peroxisome proliferator-activated receptor gamma co-activator lalpha (PGC-1alpha) and sirtuin 1 (SIRT1) reside in mitochondria: possible direct function in mitochondrial biogenesis. J Biol Chem 285: 21590-21599.

Bolte S, Cordelières FP (2006) A guided tour into subcellular colocalization analysis in light microscopy. J Microsc 224:213-232.

Chambers SM, Fasano CA, Papapetrou EP, Tomishima M, Sadelain M, Studer L (2009) Highly efficient neural conversion of human ES and iPS cells by dual inhibition of SMAD signaling. Nat Biotechnol 27:275-280.

Cui L, Jeong H, Borovecki F, Parkhurst CN, Tanese N, Krainc D (2006) Transcriptional repression of PGC-1alpha by mutant huntingtin leads to mitochondrial dysfunction and neurodegeneration. Cell 127:59-69.

Dimos JT, Rodolfa KT, Niakan KK, Weisenthal LM, Mitsumoto H, Chung W, Croft GF, Saphier G, Leibel R, Goland R, Wichterle H, Henderson CE, Eggan K (2008) Induced pluripotent stem cells generated from patients with ALS can be differentiated into motor neurons. Science 321:1218-1221.

Ebert AD, Yu J, Rose FF Jr, Mattis VB, Lorson CL, Thomson JA, Svendsen CN (2009) Induced pluripotent stem cells from a spinal muscular atrophy patient. Nature 457:277-280.

Fahn S (2003) Description of Parkinson's disease as a clinical syndrome. Ann NY Acad Sci 991:1-14.

Finck BN, Kelly DP (2006) PGC-1 coactivators: inducible regulators of energy metabolism in health and disease. J Clin Invest 116:615-622.

Gasser T (2007) Update on the genetics of Parkinson's disease. Mov Disord 22 [Suppl 17]:S343-S350.
Gegg ME, Cooper JM, Schapira AH, Taanman JW (2009) Silencing of PINK1 expression affects mitochondrial DNA and oxidative phosphorylation in dopaminergic cells. PLoS ONE 4:e4756.

Grünewald A, Breedveld GJ, Lohmann-Hedrich K, Rohé CF, König IR, Hagenah J, Vanacore N, Meco G, Antonini A, Goldwurm S, Lesage S, Dürr A, Binkofski F, Siebner H, Münchau A, Brice A, Oostra BA, Klein C, Bonifati V (2007) Biological effects of the PINK1 c.1366C > T mutation: implications in Parkinson disease pathogenesis. Neurogenetics 8:103-109.

Hedrich K, Hagenah J, Djarmati A, Hiller A, Lohnau T, Lasek K, Grünewald A, Hilker R, Steinlechner S, Boston H, Kock N, Schneider-Gold C, Kress W, Siebner H, Binkofski F, Lencer R, Münchau A, Klein C (2006) Clinical spectrum of homozygous and heterozygous PINK1 mutations in a large German family with Parkinson disease: role of a single hit? Arch Neurol 63:833-838.

Hoepken HH, Gispert S, Morales B, Wingerter O, Del Turco D, Mülsch A, Nussbaum RL, Müller K, Dröse S, Brandt U, Deller T, Wirth B, Kudin AP, Kunz WS, Auburger G (2007) Mitochondrial dysfunction, peroxidation damage and changes in glutathione metabolism in PARK6. Neurobiol Dis 25:401-411.

Huangfu D, Osafune K, Maehr R, Guo W, Eijkelenboom A, Chen S, Muhlestein W, Melton DA (2008) Induction of pluripotent stem cells from primary human fibroblasts with only Oct 4 and Sox2. Nat Biotechnol 26:1269-1275.

Kitada T, Asakawa S, Hattori N, Matsumine H, Yamamura Y, Minoshima S, Yokochi M, Mizuno Y, Shimizu N (1998) Mutations in the parkin gene cause autosomal recessive juvenile parkinsonism. Nature 392:605-608.

Klein C, Schlossmacher MG (2007) Parkinson disease, 10 years after its genetic revolution: multiple clues to a complex disorder. Neurology 69:2093-2104.

Lee G, Papapetrou EP, Kim H, Chambers SM, Tomishima MJ, Fasano CA, Ganat YM, Menon J, Shimizu F, Viale A, Tabar V, Sadelain M, Studer L (2009) Modelling pathogenesis and treatment of familial dysautonomia using patient-specific iPSCs. Nature 461:402-406.

Lee H, Shamy GA, Elkabetz Y, Schofield CM, Harrsion NL, Panagiotakos G, Socci ND, Tabar V, Studer L (2007) Directed differentiation and transplantation of human embryonic stem cell-derived motoneurons. Stem Cells 25:1931-1939.

Manders EM, Stap J, Brakenhoff GJ, van Driel R, Aten JA (1992) Dynamics of three-dimensional replication patterns during the S-phase, analysed by double labelling of DNA and confocal microscopy. J Cell Sci 103:857-862.

Narendra D, Tanaka A, Suen DF, Youle RJ (2008) Parkin is recruited selectively to impaired mitochondria and promotes their autophagy. J Cell Biol 183:795-803.

Narendra DP, Jin SM, Tanaka A, Suen DF, Gautier CA, Shen J, Cookson MR, Youle RJ (2010) PINK1 is selectively stabilized on impaired mitochondria to activate Parkin. PLoS Biol 8:e1000298.

Parfait B, Rustin P, Munnich A, Rötig A (1998) Co-amplification of nuclear pseudogenes and assessment of heteroplasmy of mitochondrial DNA mutations. Biochem Biophys Res Commun 247:57-59.

Park IH, Lerou PH, Zhao R, Huo H, Daley GQ (2008a) Generation of human-induced pluripotent stem cells. Nat Protoc 3:1180-1186.

Park IH, Arora N, Huo H, Maherali N, Ahfeldt T, Shimamura A, Lensch MW, Cowan C, Hochedlinger K, Daley GQ (2008b) Disease-specific induced pluripotent stem cells. Cell 134:877-886.

Park IH, Zhao R, West JA, Yabuuchi A, Huo H, Ince TA, Lerou PH, Lensch MW, Daley GQ (2008c) Reprogramming of human somatic cells to pluripotency with defined factors. Nature 451:141-146.

Perrier AL, Tabar V, Barberi T, Rubio ME, Bruses J, Topf N, Harrison NL, Studer L (2004) Derivation of midbrain dopamine neurons from human embryonic stem cells. Proc Natl Acad Sci U S A 101:12543-12548.

Qin W, Haroutunian V, Katsel P, Cardozo CP, Ho L, Buxbaum JD, Pasinetti GM (2009) PGC-1alpha expression decreases in the Alzheimer disease brain as a function of dementia. Arch Neurol 66:352-361.

Rakovic A, Grünewald A, Seibler P, Ramirez A, Kock N, Orolicki S, Lohmann K, Klein C (2010) Effect of endogenous mutant and wild-type PINK1 on Parkin in fibroblasts from Parkinson disease patients. Hum Mol Genet 19:3124-3137.

Soldner F, Hockemeyer D, Beard C, Gao Q, Bell GW, Cook EG, Hargus G, Blak A, Cooper O, Mitalipova M, Isacson O, Jaenisch R (2009) Parkinson's disease patient-derived induced pluripotent stem cells free of viral reprogramming factors. Cell 136:964-977. 
Takahashi K, Tanabe K, Ohnuki M, Narita M, Ichisaka T, Tomoda K, Yamanaka S (2007) Induction of pluripotent stem cells from adult human fibroblasts by defined factors. Cell 131:861-872.

Tiscornia G, Singer O, Verma IM (2006) Production and purification of lentiviral vectors. Nat Protoc 1:241-245.

Valente EM, Abou-Sleiman PM, Caputo V, Muqit MM, Harvey K, Gispert S, Ali Z, Del Turco D, Bentivoglio AR, Healy DG, Albanese A, Nussbaum R, González-Maldonado R, Deller T, Salvi S, Cortelli P, Gilks WP, Latchman DS, Harvey RJ, Dallapiccola B, et al. (2004) Hereditary early-onset Parkinson's disease caused by mutations in PINK1. Science 304:1158-1160.

Vives-Bauza C, Zhou C, Huang Y, Cui M, de Vries RL, Kim J, May J, Tocilescu MA, Liu W, Ko HS, Magrané J, Moore DJ, Dawson VL, Grailhe R, Dawson TM, Li C, Tieu K, Przedborski S (2010) PINK1dependent recruitment of Parkin to mitochondria in mitophagy. Proc Natl Acad Sci U S A 107:378-383.

Weihofen A, Ostaszewski B, Minami Y, Selkoe DJ (2008) Pink1 Parkinson mutations, the Cdc37/Hsp90 chaperones and Parkin all influence the maturation or subcellular distribution of Pink1. Hum Mol Genet 17:602-616

Weydt P, Pineda VV, Torrence AE, Libby RT, Satterfield TF, Lazarowski ER, Gilbert ML, Morton GJ, Bammler TK, Strand AD, Cui L, Beyer RP, Easley CN, Smith AC, Krainc D, Luquet S, Sweet IR, Schwartz MW, La Spada AR (2006) Thermoregulatory and metabolic defects in Huntington's disease transgenic mice implicate PGC-1alpha in Huntington's disease neurodegeneration. Cell Metab 4:349-362.

Wood-Kaczmar A, Gandhi S, Yao Z, Abramov AY, Miljan EA, Keen G,
Stanyer L, Hargreaves I, Klupsch K, Deas E, Downward J, Mansfield L, Jat P, Taylor J, Heales S, Duchen MR, Latchman D, Tabrizi SJ, Wood NW (2008) PINK1 is necessary for long term survival and mitochondrial function in human dopaminergic neurons. PLoS One 3:e2455.

Wu Z, Huang X, Feng Y, Handschin C, Feng Y, Gullicksen PS, Bare O, Labow M, Spiegelman B, Stevenson SC (2006) Transducer of regulated CREB-binding proteins (TORCs) induce PGC-1alpha transcription and mitochondrial biogenesis in muscle cells. Proc Natl Acad Sci U S A 103:14379-14384.

Xu C, Inokuma MS, Denham J, Golds K, Kundu P, Gold JD, Carpenter MK (2001) Feeder-free growth of undifferentiated human embryonic stem cells. Nat Biotechnol 19:971-974.

Xu RH, Sampsell-Barron TL, Gu F, Root S, Peck RM, Pan G, Yu J, Antosiewicz-Bourget J, Tian S, Stewart R, Thomson JA. (2008) NANOG is a direct target of TGFbeta/activin-mediated SMAD signaling in human ESCs. Cell Stem Cell 3:196-206, 2008 Aug 7.

Yao S, Sukonnik T, Kean T, Bharadwaj RR, Pasceri P, Ellis J (2004) Retrovirus silencing, variegation, extinction, and memory are controlled by a dynamic interplay of multiple epigenetic modifications. Mol Ther 10:27-36.

Zheng B, Liao Z, Locascio JJ, Lesniak KA, Roderick SS, Watt ML, Eklund AC, Zhang-James Y, Kim PD, Hauser MA, Grünblatt E, Moran LB, Mandel SA, Riederer P, Miller RM, Federoff HJ, Wüllner U, Papapetropoulos S, Youdim MB, Cantuti-Castelvetri I, et al. (2010) PGC- $1 \alpha$, a potential therapeutic target for early intervention in Parkinson's disease. Sci Transl Med 2:52ra73. 